Research Article

\title{
Motion of the Infinitesimal Variable Mass in the Generalized Circular Restricted Three-Body Problem under the Effect of Asteroids Belt
}

\author{
Ferdaous Bouaziz-Kellil ic \\ Department of Mathematics, College of Science and Arts, Qassim University, Buraydah, Saudi Arabia \\ Correspondence should be addressed to Ferdaous Bouaziz-Kellil; fm.boazuez@qu.edu.sa
}

Received 6 November 2020; Revised 24 November 2020; Accepted 30 November 2020; Published 18 December 2020

Academic Editor: Elbaz Abouelmagd

Copyright (c) 2020 Ferdaous Bouaziz-Kellil. This is an open access article distributed under the Creative Commons Attribution License, which permits unrestricted use, distribution, and reproduction in any medium, provided the original work is properly cited.

\begin{abstract}
The present paper deals with the study of the motion's properties of the infinitesimal variable mass body moving in the same orbital plan as two massive bodies (considered as primaries). It is assumed that the massive bodies have radiating effects, have oblate shapes, and are moving in circular orbits around their common center of mass. Using the procedures established by Singh and Abouelmagd, we determined the equations of motion of the infinitesimal body for which we assumed that under the effects of radiation and oblateness of the primaries, its mass varies following Jean's law. We evaluated analytically and numerically the locations of equilibrium points and examined the stability of these equilibrium points. Finally, we found that all the points are unstable.
\end{abstract}

\section{Introduction}

During the last decades, in celestial mechanics and dynamical astronomy, the most studied problem was and remains the restricted three-body problem that we denote in the sequel by R3BP. The problem has been investigated when the orbits of the primaries are either circular or elliptic. One of the reasons that make the problem very attracting is that it represents a general applicable model that can be also endowed with some types of perturbations. By perturbation, we mean the deviations of the body from its normal states due to some outer forces (perturbing effects). Perturbing effects can be in any form, such as Coriolis and centrifugal forces, different shapes of the primaries (as Roche ellipsoid, spherical shell filled with or without fluid, heterogeneous body, homogeneous body, triaxial, oblate, cylindrical, and finite straight segments), zonal harmonic effects, drag forces (P-R drag and strokes drag), resonances (high or low), solar radiation pressure, variable mass, asteroids belt, magnetic dipoles, charged bodies, Yarkovaskii effects, albedo effects, and viscous forces.

Many research studies have been devoted to this problem with different above cited perturbations. Our references are not exhaustive; however, in this introduction, we essentially cite the references that have been used to accomplish this work.

Bhatnagar and Hallan [1] introduced a new type of perturbations in the classical R3BP (i.e., under Coriolis and centrifugal forces), and they have shown that their problem has five libration points out of which three are unstable and two are stable. In their studies, Khanna and Bhatnagar [2] have been concerned by the existence and stability of equilibrium points in the circular R3BP, both with the triaxial shape and with the combination of the triaxial shape and the oblateness of the primaries. More exactly, they assumed that the more massive primary is an oblate spheroid in the first study, and in the second one, they combined the triaxial shape and the oblateness of the primaries. With similar hypothesis of the oblate spheroid shape of the more massive primary, Sharma and Subba Rao [3] investigated the stationary solutions and their characteristic exponents in the classical circular R3BP. Subba Rao and Sharma [4] studied the effect of this type of shape in the classical circular restricted three-body problem and found that the collinear stationary solutions are always unstable, while the nearly 
equilateral triangular stationary solutions are stable in some interval depending also on the oblateness factor. In the same topic of shape, Abouelmagd et al. [5] studied the effect of the oblateness associated to small perturbations in the Coriolis and centrifugal forces in R3BP. In particular, they found that the positions of the collinear points and $y$-coordinate of the triangular points are not affected by the small perturbations in the Coriolis force.

The case where both the primaries are assumed to be triaxial rigid bodies with one of their respective axes assumed to be an axis of symmetry has been investigated by Sharma et al. [6]. The authors supposed that the equatorial plane coincides with the orbital plane of motion. In these conditions, they found three collinear libration points which are always unstable and two triangular libration points which are stable in some intervals like it has been shown by Szebehely [7] for the classical restricted threebody problem. In this study, they also observed that there are long and short periodic elliptical orbits for the triangular libration points within the interval they considered. In the studies by Abouelmagd et al. [8], Ansari et al. [9], Ansari et al. [10], Ansari et al. [11], Ansari et al. [12], Ansari et al. [13], Ansari et al. [14], Ansari [15], and Ansari [16], the authors studied the models of restricted problems both in three-body, four-body, five-body, and six-body by considering various types of perturbations, especially with variable of mass. For Robe's problem, in the study by Ansari [17], the author investigated the motion of the test particle in restricted body problem having heterogeneous irregular primary filled with the viscous fluid, and in the study by Ansari et al. [10], the authors studied Robe's problem in the R3BP subject to viscous force. For the same topic, Abouelmagd et al. [18] studied Robe's problem for which they suppose that the Newton potential is subject to some modification.

On the other hand, Kushvah [19] investigated different mathematical properties due to the asteroids belts for the classical R3BP. The equilibrium points and their stability have been studied numerically. He also showed that the collinear points are unstable and the triangular points are stable in the sense of Lyapunov stability.

For the questions related to the resonance, in the study by Pathak et al. [20], the authors, in both the unperturbed and perturbed cases, investigated the location, the eccentricity, and the period of the first order exterior resonant orbits. They also analyzed the first, third, and fifth order interior resonant periodic orbits. On the other hand, the same team [21] studied resonant orbits in the framework of photogravitational planar restricted three-body problem with oblateness. It is observed that there exist periodic orbits for seventh and ninth order resonance which are passing around the Earth.

In the isotropic radiation case, the mathematical model is governed by the following data:

If $F_{1}$ and $F_{2}$ are the gravitational forces exerted on $m$ due to $m_{1}$ and $m_{2}$ and if $F_{p_{1}}$ and $F_{p_{2}}$ are the solar radiation pressure exerted on $m$ due to $m_{1}$ and $m_{2}$, respectively, then the total force exerted on $m$ due to $m_{i}$ is given by

$$
F_{i}-F_{p_{i}}=F_{i}\left(1-\frac{F_{p_{i}}}{F_{i}}\right)=F_{i}\left(1-p_{i}\right)=q_{i} F_{i}, \quad(i=1,2),
$$

where $p_{i}=$ (radiation pressure due to primary/gravitational force due to primary) and $q_{i}=\left(1-p_{i}\right), \quad 0<p_{i} \leq 1$.

Oblate body is a type of triaxial body.

$$
\frac{x^{2}}{a_{1}^{2}}+\frac{y^{2}}{b_{1}^{2}}+\frac{z^{2}}{c_{1}^{2}}=1 \text {. }
$$

When $a_{1}=b_{1}$, it will become an oblate body, and $A_{1}=$ $\left(a_{1}^{2}-c_{1}^{2}\right) / 5$ is the oblateness factor, where $a_{1}, b_{1}$, and $c_{1}$ are the semiaxes of the triaxial body [22].

Ishwar and Elipe [23] studied the generalized photogravitational R3BP where they assumed that the smaller primary is an oblate body and the massive one is the source of radiation pressure. They found secular solutions at the triangular equilibrium points, and each of these points has either a long or short periodic retrograde elliptical orbits. Singh and Taura [24] devoted their paper to the motion of an infinitesimal body in the generalized R3BP. The authors assumed that both primaries have oblate shapes, radiating and submitted to the effect of gravitational potential from a belt. They determined equations of the motion, located positions of the equilibrium points, and examined their linear stability. To the usual five equilibrium points, they showed that the corresponding problem has additional two new collinear points generated by the potential induced by the belt. They noticed that collinear points are always unstable, while triangular points are stable for certain interval of the mass ratio. Abouelmagd and Ansari [25] studied numerically the bicircular Sun perturbed Earth-Moon-satellite system and illustrated the equilibrium points, Poincarés surfaces sections, and basins of attracting domain.

In different investigations, it is always supposed that the masses of celestial bodies do not vary with time during the motion, but in reality, many celestial bodies have a variable mass with respect to the time as in the isotropic radiation or the absorption in stars. The isotropic radiation or the absorption in stars generate in general a variation of masses of these celestial bodies and constitute an interesting research topic in the celestial mechanics and dynamical astronomy. These particular last cases have been studied by many researchers in the restricted problem (two-body, three-body, four-body, five-body, and six-body).

Singh and Ishwar [26] and Lukyanov [27] investigated the effect of variable mass in the frame of circular R3BP. For their contribution, Abouelmagd and Mostafa [28] investigated the out-of-plane equilibrium points, the regions of possible motion, and the region of forbidden motion of an infinitesimal body supposed to have a variable mass relatively to Jean's law [29]. Also in R3BP, Zhang et al. [30] investigated the triangular equilibrium points when both the primaries are radiating, and the infinitesimal body has a variable mass according to Jean's law. They used Meshcherskii space-time inverse transformation [31] to test the linear stability of the equilibrium points. 
The present study can be applied to study the motion of dust particle, mass of which varies near radiating oblate binary systems surrounded by an asteroids belt.

The asteroid belts having ring shape (Figure 1) can be found in our solar system between the planets. These rings contain many bodies with irregular shapes but are always smaller than the planets themselves. In general, these asteroid belts region lies between the inner boundary (radial distance around 2.06 $\mathrm{AU}$ ) and outer boundary (the radial distance around 3.27 AU). Systems with asteroid belts were for the first time introduced by Miyamoto and Nagai [32]. This model is known as flattened potential given by the following mathematical formula:

$$
V_{b}(r, z)=\frac{M_{b}}{\left(r^{2}+\left(a+\sqrt{z^{2}+b^{2}}\right)^{2}\right)^{1 / 2}},
$$

where $M_{b}$ is the averaged mass of disc, $r$ is the radial distance of the asteroids belt from the infinitesimal body, and $a$ and $b$ are the flatness and density parameters of the asteroids belt, respectively.

Now, let us describe the organization of our paper. Section 1 presents a nonexhaustive literature review. Section 2 presents the equations of motion, while Sections 3 and 4 contain the investigations of the equilibrium points and of their stability both analytically and numerically. Finally, Section 5 represents our conclusion.

\section{Equations of Motion}

As it is commonly known, the classical R3BP is a system of three bodies of masses $m_{1}, m_{2}$, and $m$, where $m_{1}$ and $m_{2}$ represent the masses of the primaries of the system and that move in circular orbits around their common center of mass representing the origin. In our study, the primaries are assumed to be radiating with the radiation factor $q_{i}(i=1,2)$ and oblate in shape with the oblateness factor $A_{i}(i=1,2)$, respectively. In the synodic coordinate system $x y z$, the line joining both primaries are taken as the $x$-axis, while the line perpendicular to this line is known as the $y$-axis. The mean motion $n$ of the system is considered around $z$-axis, which is perpendicular to the orbital plane of the primaries. The third

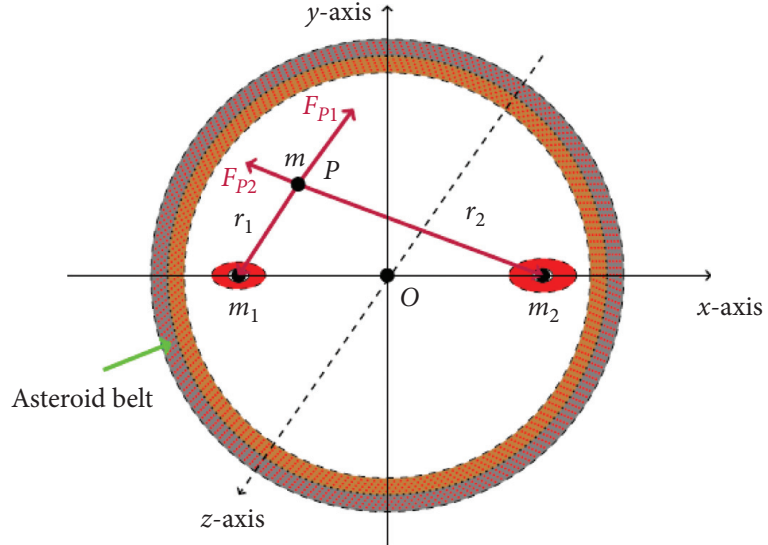

FIgURE 1: Geometric configuration of the problem with asteroid belt.

body is assumed to have an infinitesimal variable mass $m(t)$ ) and moves under the influence of the primaries and the asteroids belt of mass $M_{b}$. We also assume that this infinitesimal body does not affect the behavior of the primaries as well as the asteroids belt.

Let $r_{1}, r_{2}$, and $r$ be the distances from the infinitesimal body to the primaries $m_{1}, m_{2}$, and the asteroids belt, respectively. The coordinates of the infinitesimal body and the primaries $m_{1}$ and $m_{2}$ are denoted by $(x, y),(-\mu, 0)$, and $(1-\mu, 0)$, respectively (Figure 1$)$. Following the procedures given by Abouelmagd and Mostafa [28] and by Singh and Taura [24] and by assuming that the variation of mass of the test particle originates from one point having zero momentum, the equations of motion of the third infinitesimal variable mass $m(t)$ body with dimensionless variables in the synodic coordinate system are as follows.

$$
\left\{\begin{array}{l}
\frac{\dot{m}}{m}(\dot{x}-n y)+(\ddot{x}-2 n \dot{y})=U_{x}, \\
\frac{\dot{m}}{m}(\dot{y}+n x)+(\ddot{y}+2 n \dot{x})=U_{y},
\end{array}\right.
$$

where

$$
\left\{\begin{array}{l}
U=\frac{n^{2}}{2}\left(x^{2}+y^{2}\right)+\frac{(1-\mu) q_{1}}{r_{1}}+\frac{\mu q_{2}}{r_{2}}+\frac{(1-\mu) A_{1} q_{1}}{2 r_{1}^{3}}+\frac{\mu A_{2} q_{2}}{2 r_{2}^{3}}+\frac{M_{b}}{\sqrt{r^{2}+T^{2}}}, \\
n^{2}=1+\frac{3}{2}\left(A_{1}+A_{2}\right)+\frac{2 M_{b} r_{c}}{\left(r_{c}^{2}+T^{2}\right)^{3 / 2}},
\end{array}\right.
$$

with $\left\{\begin{array}{l}r_{1}^{2}=(x+\mu)^{2}+y^{2} \\ r_{2}^{2}=(x+\mu-1)^{2}+y^{2} \\ r^{2}=x^{2}+y^{2} \\ r_{c}^{2}=1-\mu+\mu^{2} \\ T=a+b\end{array}\right.$.

In this case, Jean's law reduces to $m=m_{0} e^{-\alpha t}$, where $\alpha$ is the constant coefficient; therefore, the mass of the body varies exponentially. Ofcourse, $m_{0}$ is the mass of the test particle at the initial time. By using the Meshcherskii spacetime transformations to preserve both space dimension and time, we get 


$$
\left\{\begin{array}{l}
x=\beta^{-1 / 2} x^{1}, \\
y=\beta^{-1 / 2} y^{1},
\end{array}\right.
$$

where $\beta=m / m_{0}$. Then, the velocity and acceleration components are as follows:

$$
\begin{aligned}
& \left\{\begin{array}{l}
\dot{x}=\beta^{-1 / 2}\left(\dot{x}^{1}+\frac{1}{2} \alpha x^{1}\right), \\
\dot{y}=\beta^{-1 / 2}\left(\dot{y}^{1}+\frac{1}{2} \alpha y^{1}\right) .
\end{array}\right. \\
& \left\{\begin{array}{l}
\ddot{x}=\beta^{-1 / 2}\left(\ddot{x}^{1}+\alpha \dot{x}^{1}+\frac{1}{4} \alpha^{2} x^{1}\right), \\
\ddot{y}=\beta^{-1 / 2}\left(\ddot{y}^{1}+\alpha \dot{y}^{1}+\frac{1}{4} \alpha^{2} y^{1}\right) .
\end{array}\right.
\end{aligned}
$$

After using equations 6-8, equation (4) becomes

$$
\left\{\begin{array}{l}
\ddot{x}^{1}-2 n \dot{y}^{1}=V_{x^{1}}, \\
\ddot{y}^{1}+2 n \dot{x}^{1}=V_{y^{1}},
\end{array}\right.
$$

where

$$
\begin{aligned}
V= & \left(\frac{n^{2}}{2}+\frac{\alpha^{2}}{8}\right)\left(\left(x^{1}\right)^{2}+\left(y^{1}\right)^{2}\right) \\
& +\beta^{3 / 2}\left(\frac{(1-\mu) q_{1}}{\rho_{1}}+\frac{\mu q_{2}}{\rho_{2}}+\frac{(1-\mu) q_{1} A_{1} \beta}{2 \rho_{1}^{3}}+\frac{\mu q_{2} A_{2} \beta}{2 \rho_{2}^{3}}+\frac{M_{b}}{\sqrt{\rho^{2}+T^{2} \beta}}\right) .
\end{aligned}
$$

$\rho_{1}, \rho_{2}$, and $\rho$ are defined by

$$
\left\{\begin{array}{l}
\rho_{1}^{2}=\left(x^{1}+\sqrt{\beta} \mu\right)^{2}+\left(y^{1}\right)^{2}, \\
\rho_{2}^{2}=\left(x^{1}+\sqrt{\beta} \mu-\sqrt{\beta}\right)^{2}+\left(y^{1}\right)^{2}, \\
\rho^{2}=\left(x^{1}\right)^{2}+\left(y^{1}\right)^{2} .
\end{array}\right.
$$

\section{Analysis of Equilibrium Points}

If we replace the derivative with respect to time on the left hand side of system (9) by zero, we get

$$
\begin{aligned}
& x^{1}\left(\frac{\alpha^{2}}{4}+n^{2}\right)+\beta^{3 / 2}\left(-\frac{q_{1}(1-\mu)\left(x^{1}+\sqrt{\beta} \mu\right)}{\rho_{1}^{3}}-\frac{q_{2}\left(x^{1}+\sqrt{\beta}(-1+\mu)\right) \mu}{\rho_{2}^{3}}-\frac{3 A_{1} q_{1} \beta(1-\mu)\left(x^{1}+\sqrt{\beta} \mu\right)}{2 \rho_{1}^{5}}\right. \\
& \left.\quad-\frac{3 A_{2} q_{2} \beta\left(x^{1}+\sqrt{\beta}(-1+\mu)\right) \mu}{2 \rho_{2}^{5}}-\frac{M_{b} x^{1}}{\left(\rho^{2}+T^{2} \beta\right)^{3 / 2}}\right)=0, \\
& y^{1}\left(\frac{\alpha^{2}}{4}+n^{2}\right)+y^{1} \beta^{3 / 2}\left(-\frac{q_{1}(1-\mu)}{\rho_{1}^{3}}-\frac{q_{2} \mu}{\rho_{2}^{3}}-\frac{3 A_{1} q_{1} \beta(1-\mu)}{2 \rho_{1}^{5}}-\frac{3 A_{2} q_{2} \beta \mu}{2 \rho_{2}^{5}}-\frac{M_{b}}{\left(\rho^{2}+T^{2} \beta\right)^{3 / 2}}\right)=0 . \\
& \text { lar Equilibrium Points. From equations (12) and } \quad \text { (14) } \frac{\alpha^{2}}{4}+n^{2}-\beta^{3 / 2}\left(\frac{q_{1}}{\rho_{1}^{3}}+\frac{3 A_{1} q_{1} \beta}{2 \rho_{1}^{5}}+\frac{M_{b}}{\left(\rho^{2}+T^{2} \beta\right)^{3 / 2}}\right)=0, \\
& \frac{q_{1}}{\rho_{1}^{3}}+\frac{3 A_{1} q_{1} \beta}{2 \rho_{1}^{5}}=\frac{q_{2}}{\rho_{2}^{3}}+\frac{3 A_{2} q_{2} \beta}{2 \rho_{2}^{5}} \text {. }
\end{aligned}
$$

Taking in account equation (14), equations (12) and (13) can be written, respectively, as

In the classical R3BP (i.e., when $\alpha=0, \beta=1, q_{i}=0$, and $\left.A_{i}=0\right)$, the solution is $\left(\rho_{1}=1, \rho_{2}=1\right)$. Therefore, let us consider that the solution in our problem is $\left(\rho_{1}=1+\gamma_{1}, \rho_{2}=\right.$ $1+\gamma_{2}$ ), where $\gamma_{1} \ll 1$ and $\gamma_{2} \ll 1$. From (15) and (16), we get

$$
\begin{aligned}
& \gamma_{1}=\frac{1}{3}-\frac{p_{1}}{3}\left(1+\frac{\alpha^{2}}{4}\right) \beta^{(-3 / 2)}+\frac{A_{1}}{2}\left(1+\frac{\alpha^{2}}{4}\right) \beta^{(-1 / 2)}-\frac{1}{3}\left(n^{2}+\frac{\alpha^{2}}{4}\right) \beta^{(-3 / 2)}+\frac{M_{b}}{3\left(\rho^{2}+T^{2} \beta\right)^{3 / 2}} \\
& \gamma_{2}=\frac{1}{3}-\frac{p_{2}}{3}\left(1+\frac{\alpha^{2}}{4}\right) \beta^{(-3 / 2)}+\frac{A_{2}}{2}\left(1+\frac{\alpha^{2}}{4}\right) \beta^{(-1 / 2)}-\frac{1}{3}\left(n^{2}+\frac{\alpha^{2}}{4}\right) \beta^{(-3 / 2)}+\frac{M_{b}}{3\left(\rho^{2}+T^{2} \beta\right)^{3 / 2}} .
\end{aligned}
$$


And from system (11), we get

By combining equations (15-19), we obtain

$$
\left\{\begin{array}{l}
x^{1}=\sqrt{\beta}\left(\frac{1}{2}-\mu\right)+\frac{1}{\sqrt{\beta}}\left(\gamma_{1}-\gamma_{2}\right), \\
y^{1}= \pm \frac{\sqrt{4-\beta}}{2}\left(1+\frac{2}{(4-\beta)}\left(\gamma_{1}+\gamma_{2}\right)\right) .
\end{array}\right.
$$

$$
\left\{\begin{array}{l}
x^{1}=\sqrt{\beta}\left(\frac{1}{2}-\mu\right)-\frac{1}{\beta}\left(1+\frac{\alpha^{2}}{4}\right)\left(\frac{p_{1}-p_{2}}{3 \beta}-\frac{A_{1}-A_{2}}{2}\right), \\
y^{1}= \pm \frac{\sqrt{4-\beta}}{2}\left[1+\frac{2}{(4-\beta)}\left(\frac{2}{3}-\frac{p_{1}+p_{2}}{3}\left(1+\frac{\alpha^{2}}{4}\right) \beta^{(-3 / 2)}+\frac{A_{1}+A_{2}}{2}\left(1+\frac{\alpha^{2}}{4}\right) \beta^{(-1 / 2)}-\frac{2}{3}\left(n^{2}+\frac{\alpha^{2}}{4}\right) \beta^{(-3 / 2)}+\frac{2 M_{b}}{3\left(\rho^{2}+T^{2} \beta\right)^{(3 / 2)}}\right)\right] .
\end{array}\right.
$$

Notice that equation (20) represents the coordinates of triangular equilibrium points.
3.2. Collinear Equilibrium Points. In this subsection and from equation (12), we will determine the collinear equilibrium points. By replacing $y^{1}$ by 0 in equation (12), we get

$$
\begin{aligned}
f\left(x^{1}, y^{1}\right)= & x^{1}\left(\frac{\alpha^{2}}{4}+n^{2}\right)+\beta^{3 / 2}\left(-\frac{q_{1}(1-\mu)\left(x^{1}+\sqrt{\beta} \mu\right)}{\rho_{1}^{3}}-\frac{q_{2}\left(x^{1}+\sqrt{\beta}(-1+\mu)\right) \mu}{\rho_{2}^{3}}-\frac{3 A_{1} q_{1} \beta(1-\mu)\left(x^{1}+\sqrt{\beta} \mu\right)}{2 \rho_{1}^{5}}\right. \\
& \left.-\frac{3 A_{2} q_{2} \beta\left(x^{1}+\sqrt{\beta}(-1+\mu)\right) \mu}{2 \rho_{2}^{5}}-\frac{M_{b} x^{1}}{\left(\rho^{2}+T^{2} \beta\right)^{3 / 2}}\right),
\end{aligned}
$$

and therefore,

$$
f\left(x^{1}, 0\right)=s_{1}\left(x^{1}\right)+s_{2}\left(x^{1}\right),
$$

where

$$
\begin{aligned}
s_{1}\left(x^{1}\right)= & x^{1}\left(\frac{\alpha^{2}}{4}+n^{2}\right)+\beta^{3 / 2}\left(-\frac{q_{1}(1-\mu)\left(x^{1}+\sqrt{\beta} \mu\right)}{\left|x^{1}+\sqrt{\beta} \mu^{3}\right|}-\frac{q_{2}\left(x^{1}+\sqrt{\beta}(-1+\mu)\right) \mu}{\left|x^{1}+\sqrt{\beta}(-1+\mu)^{3}\right|}-\frac{3 A_{1} q_{1} \beta(1-\mu)\left(x^{1}+\sqrt{\beta} \mu\right)}{2 \mid x^{1}+\sqrt{\beta} \mu^{5}}\right. \\
& \left.-\frac{3 A_{2} q_{2} \beta\left(x^{1}+\sqrt{\beta}(-1+\mu)\right) \mu}{2\left|x^{1}+\sqrt{\beta}(-1+\mu)\right|^{5}}\right), \\
s_{2}\left(x^{1}\right)= & -\frac{M_{b} x^{1} \beta^{3 / 2}}{\left(\rho^{2}+T^{2} \beta\right)^{3 / 2}} .
\end{aligned}
$$

To determine the locations of collinear equilibrium points, we divide the $x$-axis in three different subintervals, that is, $\quad x^{1} \in(-\infty,-\mu \sqrt{\beta}), \quad x^{1} \in$ $(-\mu \sqrt{\beta},(1-\mu) \sqrt{\beta})$, and $x^{1} \in((1-\mu) \sqrt{\beta}, \infty)$, and we will specify our approach in each case separately. Notice that the endpoints of the above intervals correspond to the situations where the infinitesimal body coincides with one of the primaries. 
3.2.1. First Case. For the interval $x^{1} \in(-\infty,-\mu \sqrt{\beta})$,

$$
\begin{aligned}
s_{1}\left(x^{1}\right) & =x^{1}\left(\frac{\alpha^{2}}{4}+n^{2}\right)+\beta^{3 / 2}\left(\frac{q_{1}(1-\mu)}{\left(x^{1}+\sqrt{\beta} \mu\right)^{2}}+\frac{q_{2} \mu}{\left(x^{1}+\sqrt{\beta}(-1+\mu)\right)^{2}}+\frac{3 A_{1} q_{1} \beta(1-\mu)}{2\left(x^{1}+\sqrt{\beta} \mu\right)^{4}}+\frac{3 A_{2} q_{2} \beta \mu}{2\left(x^{1}+\sqrt{\beta}(-1+\mu)\right)^{4}}\right), \\
s_{1}^{\prime}\left(x^{1}\right) & =n^{2}+\frac{\alpha^{2}}{4}+\beta^{(3 / 2)}\left(-\frac{2 q_{1}(1-\mu)}{\left(x^{1}+\sqrt{\beta} \mu\right)^{3}}-\frac{2 q_{2} \mu}{\left(x^{1}+\sqrt{\beta}(-1+\mu)\right)^{3}}-\frac{6 A_{1} q_{1} \beta(1-\mu)}{\left(x^{1}+\sqrt{\beta} \mu\right)^{5}}-\frac{6 A_{2} q_{2} \beta \mu}{\left(x^{1}+\sqrt{\beta}(-1+\mu)\right)^{5}}\right) \\
& =n^{2}+\frac{\alpha^{2}}{4}+\beta^{(3 / 2)}\left(\frac{2 q_{1}(1-\mu)}{\left|x^{1}+\sqrt{\beta} \mu\right|^{3}}+\frac{2 q_{2} \mu}{\left|x^{1}+\sqrt{\beta}(-1+\mu)\right|^{3}}+\frac{6 A_{1} q_{1} \beta(1-\mu)}{\left|x^{1}+\sqrt{\beta} \mu\right|^{5}}+\frac{6 A_{2} q_{2} \beta \mu}{\left|x^{1}+\sqrt{\beta}(-1+\mu)\right|^{5}}\right) .
\end{aligned}
$$

It is clear that $s_{1}^{\prime}\left(x^{1}\right)>0$ for $x^{1} \in(-\infty,-\mu \sqrt{\beta})$, and $s_{1}\left(x^{1}\right)$ is then a monotonically increasing function and $\lim _{x^{1} \longrightarrow-\infty} s_{1}\left(x^{1}\right)=-\infty$, and $\lim _{x^{1} \longrightarrow-\sqrt{\beta} \mu^{-}} s_{1}\left(x^{1}\right)=\infty$.

We also have $s_{2}\left(x^{1}\right)=M_{b}\left|x^{1}\right| \beta^{3 / 2} /\left(\left(x^{1}\right)^{2}+T^{2} \beta\right)^{3 / 2}$, and $s_{2}^{\prime}\left(x^{1}\right)=M_{b} \beta^{(3 / 2)} /\left(\left(x^{1}\right)^{2}+T^{2} \beta\right)^{(3 / 2)}-3 M_{b}\left(x^{1}\right)^{2} \beta^{(3 / 2)} /$ $\left(\left(x^{1}\right)^{2}+T^{2} \beta\right)^{(5 / 2)}<0 . \quad \lim _{x^{1} \longrightarrow-\infty} s_{2}\left(x^{1}\right)=0, \quad$ and $\lim _{x^{1} \longrightarrow-\sqrt{\beta} \mu^{-}} s_{2}\left(x^{1}\right)>0, s_{2}\left(x^{1}\right)$ is a monotonically increasing function.

As $\lim _{x^{1} \longrightarrow-\infty} f\left(x^{1}, 0\right)<0$ and $\quad \lim _{x^{1}} \longrightarrow-\sqrt{\beta} \mu^{-}$ $f\left(x^{1}, 0\right)>0$, we can conclude that there exists a unique real in the interval $x^{1} \in(-\infty,-\mu \sqrt{\beta})$ for which $f\left(x^{1}, 0\right)=0$, and the corresponding point will be denoted in the sequel by $L_{3}$.

3.2.2. Second Case. For the case where $x^{1} \in(-\mu \sqrt{\beta},(1-\mu) \sqrt{\beta})=(-\mu \sqrt{\beta}, 0) \cup(0,(1-\mu) \sqrt{\beta})$, we will treat in the first step the subcase when $x^{1} \in(-\mu \sqrt{\beta}, 0)=(-\mu \sqrt{\beta},-T \sqrt{\beta} / \sqrt{2}) \cup((-T \sqrt{\beta}) / \sqrt{2}, 0)$.

Let $x^{1} \in(-\mu \sqrt{\beta},-T \sqrt{\beta} / \sqrt{2})$. Since $\lim _{x^{1}} \longrightarrow-\sqrt{\beta} \mu^{+}$ $s_{1}\left(x^{1}\right)=-\infty$ and $\lim _{x^{1} \longrightarrow-\sqrt{\beta} \mu^{+}} s_{2}\left(x^{1}\right)>0$, we get $\lim _{x^{1} \longrightarrow-\sqrt{\beta} \mu^{+}} f\left(x^{1}, 0\right)<0, \quad$ and $s_{1}((-T \sqrt{\beta}) / \sqrt{2})+$ $s_{2}((-T \sqrt{\beta}) / \sqrt{2})>0$. Consequently, $f((-T \sqrt{\beta}) / \sqrt{2}, 0)>0$, which means there is a unique point for which $f\left(x^{1}, 0\right)=0$. This point will be denoted in the sequel by $L_{b 1}$.

In the case where $x^{1} \in((-T \sqrt{\beta}) / \sqrt{2}, 0)$, we have $s_{1}((-T \sqrt{\beta}) / \sqrt{2})+s_{2}((-T \sqrt{\beta}) / \sqrt{2})>0$, which implies that $f((-T \sqrt{\beta}) / \sqrt{2}, 0)>0$ and $f(0,0)=\beta^{3 / 2}\left(-q_{1}(1-\mu)\right.$ $(\sqrt{\beta} \mu) /(\sqrt{\beta} \mu)^{3}-q_{2}(\sqrt{\beta}(-1+\mu)) \mu /(\sqrt{\beta}(-1+\mu))^{3}-3 A_{1} q_{1}$ $\beta(1-\mu)(\sqrt{\beta} \mu) / 2(\sqrt{\beta} \mu)^{5}-3 A_{2} q_{2} \beta(\sqrt{\beta}(-1+\mu)) \mu / 2(\sqrt{\beta}$ $\left.(-1+\mu))^{5}\right)<0$
We can interpret as above that there exists a unique point for which $f\left(x^{1}, 0\right)=0$. Let us denote this point by $L_{b 2}$.

To complete the study of this second case, let $x^{1} \in(0,(1-\mu) \sqrt{\beta})$.

Since $f(0,0)<0$ and $\lim _{x^{1}} \longrightarrow \sqrt{\beta}(1-\mu)^{-} f\left(x^{1}, 0\right)>0$, we can conclude that there exists a unique point for which $f\left(x^{1}, 0\right)=0$. Let $L_{2}$ be this point.

3.2.3. Third Case. Let $x^{1} \in((1-\mu) \sqrt{\beta}, \infty)$. Since $\lim _{x^{1} \longrightarrow \sqrt{\beta}(1-\mu)^{+}} s_{1}\left(x^{1}\right)=-\infty, \quad \lim _{x^{1} \longrightarrow \infty} s_{1}\left(x^{1}\right)=\infty$, $s_{2}(\sqrt{\beta}(1-\mu))<0$, and $\lim _{x^{1}} \longrightarrow \infty s_{2}\left(x^{1}\right)=0$, we can conclude that $\lim _{x^{1} \longrightarrow \sqrt{\beta}(1-\mu)^{+}} f\left(x^{1}, 0\right)<0$ and $\lim _{x^{1} \rightarrow \infty} f\left(x^{1}, 0\right)>0$, and then, we conclude that there is a unique real in this interval for which $f\left(x^{1}, 0\right)=0$. The corresponding point will be denoted by $L_{1}$.

The above points $L_{1}, L_{2}, L_{3}, L_{b 1}$, and $L_{b 2}$ are called collinear equilibrium points (Figure 2 and 3 ). These points are similar to points determined in the study by Singh and Taura [24]. Notice that in the classical R3BP, there are only three collinear equilibrium points.

The locations of these equilibrium points are determined numerically and depicted in Figure 4. From analyzing this figure, we can observe that as we increase the value of $\beta$, all the equilibrium points are moving away from the origin except $L_{b 2}$ (Figure 5).

\section{Stability of Equilibrium Points}

In this section, let us investigate the stability properties of the small body's motion in its vicinity $\left(x^{10}+x^{11}, y^{10}+y^{11}\right)$ under the effect of the oblate radiating primaries and the asteroids dust belt, where $\left(x^{11}, y^{11}\right)$ are the small displacements from the equilibrium points $\left(x^{10}, y^{10}\right)$. To do this, we can write the variational equations for system (9) as

$$
\left\{\begin{array}{l}
\ddot{x}^{11}-2 n \dot{y}^{11}=V_{x^{1} x^{1}}^{0} x^{11}+V_{x^{1} y^{1}}^{0} y^{11} \\
\ddot{y}^{11}+2 n \dot{x}^{11}=V_{y^{1} x^{1}}^{0} x^{11}+V_{y^{1} x^{1}}^{0} y^{11}
\end{array}\right.
$$




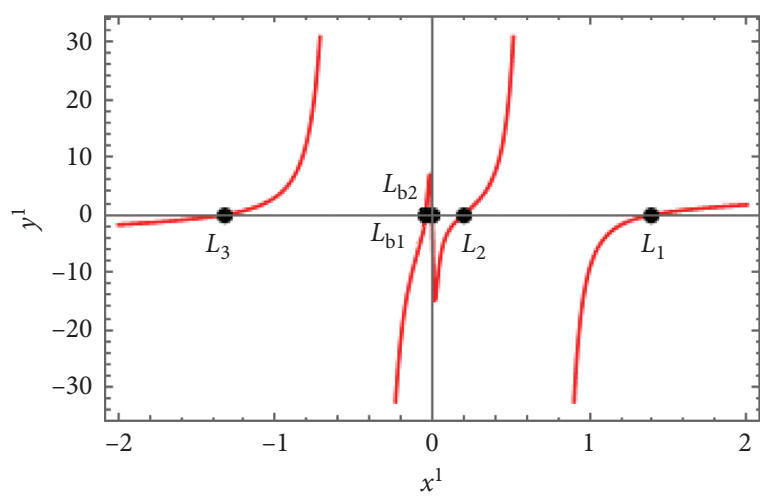

FIgURe 2: Locations of collinear equilibrium points.

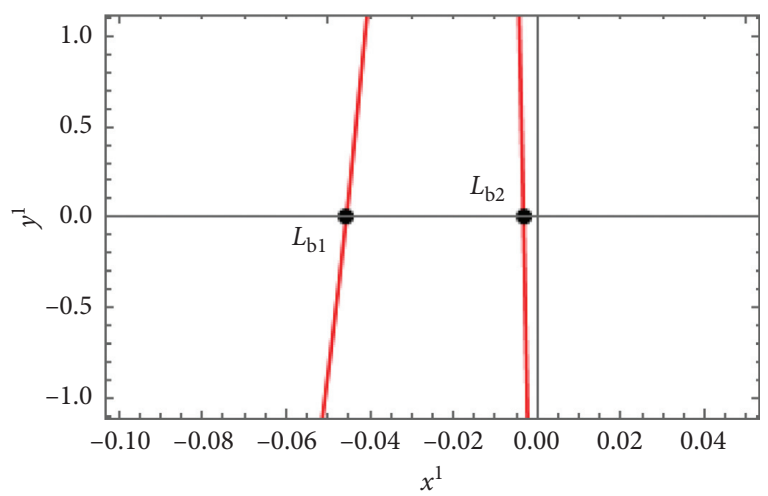

FIGURE 3: Zoomed part of Figure 2 near $L_{b 2}$.

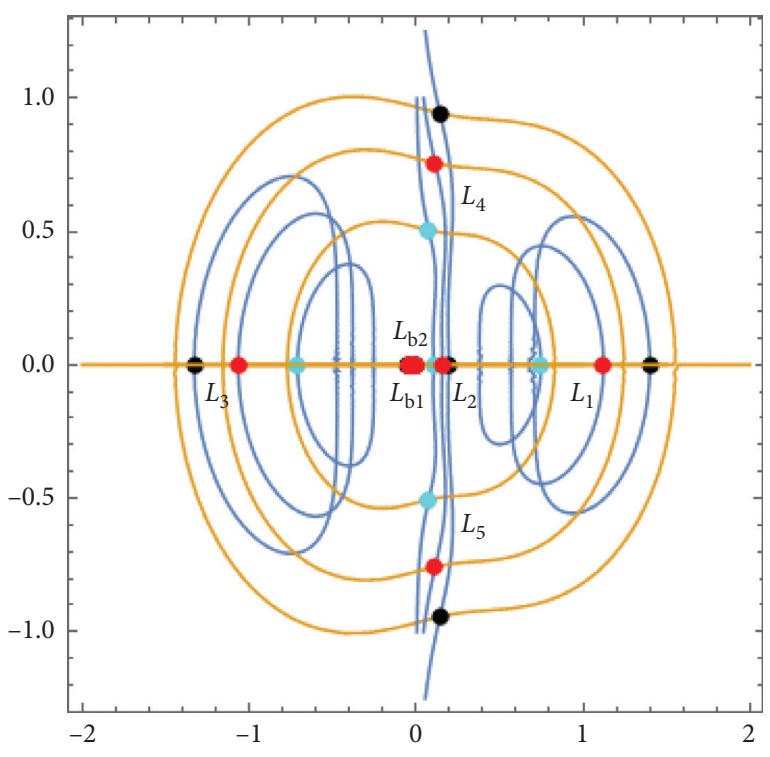

Figure 4: Locations of all equilibrium points for three values of $\beta$ (0.4 (cyan), 0.9 (red), and 1.4 (black)).

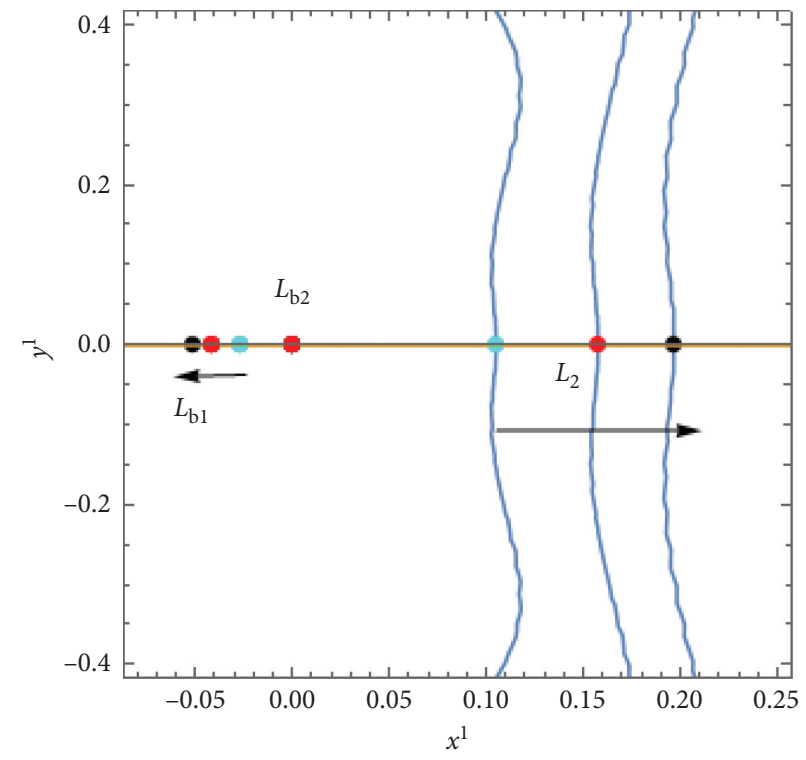

FIGURE 5: Zoomed part of Figure 4 near $L_{b 2}$ and their movement.

The superscript 0 denotes the value at the corresponding equilibrium point.

In the phase space, the above system (25) can be rewritten as

$$
\left\{\begin{array}{l}
\dot{x}=x^{12}, \\
\dot{y}^{11}=y^{12}, \\
\dot{x}^{12}=2 n y^{12}+V_{x^{1} x^{1}}^{0} x^{11}+V_{x^{1} y^{1}}^{0} y^{11}, \\
\dot{y}^{12}=-2 n x^{12}+V_{y^{1} x^{1}}^{0} x^{11}+V_{y^{1} y^{1}}^{0} y^{11} .
\end{array}\right.
$$

Due to the variation of the mass and of the distance of the small particle, by using Meshcherskii space-time inverse transformations to examine the stability of the equilibrium points, we then get

$$
\left\{\begin{array}{l}
x^{13}=\beta^{-1 / 2} x^{11}, y^{13}=\beta^{-1 / 2} y^{11}, \\
x^{14}=\beta^{-1 / 2} x^{12}, y^{14}=\beta^{-1 / 2} y^{12} .
\end{array}\right.
$$

Taking in account equation (26), system (27) can be written as follows:

$$
\dot{Y}=B \mathbf{Y},
$$

where

$$
Y=\left(\begin{array}{c}
x^{13} \\
y^{13} \\
x^{14} \\
y^{14}
\end{array}\right),
$$


TABle 1: All the equilibrium points depicted are unstable and determined for $T=0.02, q_{1}=0.90, q_{2}=0.85, A_{1}=0.03, A_{2}=0.02$, $M_{b}=0.01, \mu=0.4$, and $\alpha=0.2$.

\begin{tabular}{|c|c|c|c|}
\hline $\begin{array}{l}\text { Equil } \\
\beta\end{array}$ & $x^{1}-C_{0}$ & $y^{1}-C_{0}$ & Roots \\
\hline \multirow{6}{*}{0.40} & 0.7482467178 & 0.0000000000 & $\begin{array}{c}0.1 \pm 1.46479 i \\
1.6105 \\
-1.4105\end{array}$ \\
\hline & 0.1051524050 & 0.0000000000 & $\begin{array}{c}0.1 \pm 3.25487 i \\
-4.46466 \\
4.66466\end{array}$ \\
\hline & -0.7099161991 & 0.0000000000 & $\begin{array}{c}0.1 \pm 1.30658 i \\
-1.08426 \\
1.28426\end{array}$ \\
\hline & -0.0274388781 & 0.000000000 & $\begin{array}{c}0.1 \pm 10.4769 i \\
-13.2108 \\
13.4108\end{array}$ \\
\hline & -0.0002097310 & 0.0000000000 & $\begin{array}{l}0.1 \pm 36.3091 i \\
0.1 \pm 34.1204 i\end{array}$ \\
\hline & 0.0766012649 & \pm 0.5044421565 & $\begin{array}{c}-0.610786 \pm 0.997642 i \\
0.810786 \pm 0.997642 i\end{array}$ \\
\hline \multirow{7}{*}{0.90} & 1.1223700767 & 0.0000000000 & $\begin{array}{c}0.1 \pm 1.46479 i \\
-1.4105 \\
1.6105\end{array}$ \\
\hline & 0.1577286075 & 0.0000000000 & $\begin{array}{c}0.1 \pm 3.25487 i \\
\pm 4.46466 \\
0.1 \pm 1.30658 i\end{array}$ \\
\hline & -1.0648742987 & 0.0000000000 & -1.08426 \\
\hline & & & 1.28426 \\
\hline & -0.0411583172 & 0.0000000000 & $\begin{array}{c}0.1 \pm 10.4769 i \\
-13.2108 \\
13.4108\end{array}$ \\
\hline & -0.0003146670 & 0.0000000000 & $\begin{array}{l}0.1 \pm 34.1204 i \\
0.1 \pm 36.3091 i\end{array}$ \\
\hline & 0.1149018973 & \pm 0.7566632347 & $\begin{array}{c}-0.610786 \pm 0.997642 i \\
0.810786 \pm 0.997642 i\end{array}$ \\
\hline \multirow{6}{*}{1.40} & 1.3998414294 & 0.0000000000 & $\begin{array}{c}0.1 \pm 1.46479 i \\
-1.4105 \\
1.6105\end{array}$ \\
\hline & 0.1967221364 & 0.0000000000 & $\begin{array}{c}0.1 \pm 3.25487 i \\
-4.46466 \\
4.66466\end{array}$ \\
\hline & -1.3281315953 & 0.0000000000 & $\begin{array}{c}0.1 \pm 1.30658 i \\
-1.08426 \\
1.28426\end{array}$ \\
\hline & -0.0513334406 & 0.0000000000 & $\begin{array}{c}0.1 \pm 10.4769 i \\
-13.2108 \\
13.4108\end{array}$ \\
\hline & -0.0003923708 & 0.0000000000 & $\begin{array}{l}0.1 \pm 34.1204 i \\
0.1 \pm 36.3091 i\end{array}$ \\
\hline & 0.1433078443 & \pm 0.9437248605 & $\begin{array}{l}0.610786 \pm 0.997642 i \\
0.810786 \pm 0.997642 i\end{array}$ \\
\hline
\end{tabular}




$$
B=\left(\begin{array}{cccc}
\frac{1}{2} \alpha & 0 & 1 & 0 \\
0 & \frac{1}{2} \alpha & 0 & 1 \\
V_{x^{1} x^{1}}^{0} & V_{x^{1} y^{1}}^{0} & \frac{1}{2} \alpha & 2 n \\
V_{y^{1} x^{1}}^{0} & V_{y^{1} y^{1}}^{0} & -2 n & \frac{1}{2} \alpha
\end{array}\right) .
$$

The characteristic equation for the matrix $B$ is then

$$
\lambda^{4}+\alpha_{3} \lambda^{3}+\alpha_{2} \lambda^{2}+\alpha_{1} \lambda+\alpha_{0}=0
$$

where

$$
\left\{\begin{array}{l}
\alpha_{0}=\frac{1}{16} \alpha^{4}+\frac{1}{4} \alpha^{2}\left(4 n^{2}-V_{x^{1} x^{1}}-V_{y^{1} y^{1}}\right)+\left(V_{x^{1} x^{1}} V_{y^{1} y^{1}}-V_{x^{1} y^{1}}^{2}\right), \\
\alpha_{1}=\alpha\left(V_{x^{1} x^{1}}+V_{y^{1} y^{1}}-4 n^{2}-\frac{\alpha^{2}}{2}\right), \\
\alpha_{2}=-\left(V_{x^{1} x^{1}}+V_{y^{1} y^{1}}-4 n^{2}-\frac{3 \alpha^{2}}{2}\right), \\
\alpha_{3}=-2 \alpha .
\end{array}\right.
$$

Table 1 represents the numerical solutions of equation (30), for the values $T=0.02, q_{1}=0.90, q_{2}=0.85, A_{1}=0.03$, $A_{2}=0.02, M_{b}=0.01, \mu=0.4$, and $\alpha=0.2$ (Singh and Taura [24] and Ansari [16]) and three different values of parameter. This table represents also the roots corresponding to each equilibrium points. From a simple interpretation of the results of the table, we can deduce that equilibrium points are unstable because at least one characteristic root is either a positive real number or positive real part of the complex characteristic root. While in the study by Singh and Taura [24], it is shown that some equilibrium points are stable in some intervals; therefore, in our case due to the impact of the variation parameters, all the equilibrium points obtained are unstable.

\section{Conclusion}

In this paper, we studied the effects of the variation parameters $\alpha$ and $\beta$ on the behavior of motion of the infinitesimal body in the restricted 3-body problem and also when the mass of this infinitesimal body varies according to Jean's law. We assumed that the primaries have both radiating as well as oblateness effects, and the whole system has an effect of an asteroids belt. Using the Meshcherskii space-time transformation, we have evaluated the equations of motion. From the obtained system of equations of motion, we numerically illustrated the seven equilibrium points where five equilibrium points are collinear and two are noncollinear (i.e., triangular equilibrium points). This conclusion is similar to that made by Singh and Taura [24] but more different from the classical R3BP [7]. Figure 4 shows the location of the seven equilibrium points and their movements for three values of $\beta(0.4,0.9$, and 1.4$)$. Figure 5 is the zoomed part of Figure 4 near the equilibrium point $L_{b 2}$. From these figures, we noticed that as we increase the value of the variation parameter $\beta$, all the equilibrium points are moving away from the origin except $L_{b 2}$. Furthermore, we examined the stability of equilibrium points numerically, and Table 1 represents the roots of the characteristic polynomial that shows that at least one of the roots has either positive real part of the complex roots or only a positive real root. These facts confirm that all the equilibrium points are unstable. As a second remark, we deduced that this result is different from the result obtained by Singh and Taura [24] where they have shown that the triangular points are stable for $0<\mu<\mu_{c}$, where $\mu_{c}$ is the critical mass ratio influenced by the oblateness and radiation parameters of the primaries and potential from the belt. We can then conclude that the variation of parameters has a great impact on the dynamical behavior of the motion of the infinitesimal body.

\section{Data Availability}

The data depicted in the table are used to support the findings of this study are included within the article.

\section{Conflicts of Interest}

The author declares that there are no conflicts of interest.

\section{Acknowledgments}

The author is thankful to the Deanship of Scientific Research, College of Science at Buraidha, Qassim University, Saudi Arabia, for providing all the research facilities in the completion of this research work. The author wishes to express his sincere thanks to referees who provided precious expertise that greatly helped to improve the paper.

\section{References}

[1] K. B. Bhatnagar and P. P. Hallan, "Effect of perturbed potentials on the stability of libration points in the restricted problem," Celestial Mechanics, vol. 20, no. 2, pp. 95-103, 1979.

[2] M. Khanna and K. B. Bhatnagar, "Existence and stability of libration points in the restricted three-body problem when the smaller primary is a triaxial rigid body and the bigger one an oblate spheroid," Indian Journal of Pure and Applied Mathematics (IJPAM), vol. 30, no. 7, pp. 721-733, 1999.

[3] R. K. Sharma and P. V. Subba Rao, "Stationary solutions and their characteristic exponents in the restricted three-body problem when the more massive primary is an oblate spheroid," Celestial Mechanics and Dynamical Astronomy, vol. 13, no. 2, pp. 137-149, 1976.

[4] P. V. Subba Rao and R. K. Sharma, "A note on the stability of the triangular points of the equilibrium in the restricted threebody problem," Astronomy and Astrophysics, vol. 43, no. 3, pp. 381-383, 1975.

[5] E. I. Abouelmagd, H. M. Asiri, and M. A. Sharaf, "The effect of oblateness in the perturbed restricted three-body problem," Meccanica, vol. 48, no. 10, pp. 2479-2490, 2013. 
[6] R. K. Sharma, Z. A. Taqvi, and K. B. Bhatnagar, "Existence and stability of libration points in the restricted three-body problem when the primaries are triaxial rigid bodies," $\mathrm{Ce}$ lestial Mechanics and Dynamical Astronomy, vol. 79, no. 2, pp. 119-133, 2001.

[7] V. Szebehely, Theory of Orbits, Academic Press, NY, USA, 1967.

[8] E. I. Abouelmagd, A. A. Ansari, M. S. Ullah, and J. L. García Guirao, "A planar five-body problem in a framework of heterogeneous and mass variation effects," The Astronomical Journal, vol. 160, no. 5, p. 216, 2020.

[9] A. A. Ansari, K. R. Meena, and S. N. Prasad, "Perturbed sixbody configuration with variable mass," Romanian Astronomical Journal, vol. 30, no. 2, pp. 135-152, 2020.

[10] A. A. Ansari, J. Singh, Z. A. Alhussain, and H. Belmabrouk, "Perturbed Robe's CR3BP with viscous force," Astrophysics and Space Science, vol. 364, no. 6, p. 95, 2019.

[11] A. A. Ansari, R. Kellil, Z. A. Al-Hussain, and W. Ul-Haq, "Effect of variation of charge in the circular restricted threebody problem with variable masses," Journal of Taibah University for Science, vol. 13, no. 1, pp. 670-677, 2019.

[12] A. A. Ansari, R. Kellil, and Z. A. Al-Hussain, "Behavior of an infinitesimal-variable-mass body in CR3BP; the primaries are finite straight segments," Punjab University Journal of Mathematics, vol. 51, no. 5, pp. 107-120, 2019.

[13] A. A. Ansari, K. Shalini, and Z. A. Alhussain, "Non-linear stability of $L_{4}$ in the R3BP when the smaller primary is a heterogeneous triaxial rigid body with $\mathrm{N}$ layers," Italian Journal of Pure and Applied Mathematics, vol. 41, pp. 297-312, 2019.

[14] A. A. Ansari, A. Ali, M. Alam, and R. Kellil, "Cyclic kite configuration with variable mass of the fifth body in R5BP," Application and Applied Mathematics: An International Journal (AAM), vol. 14, no. 2, pp. 985-1002, 2019.

[15] A. A. Ansari, "The circular restricted four- body problem with triaxial primaries and variable infinitesimal mass," Applications and Applied Mathematics: An International Journal, vol. 13, no. 2, pp. 818-838, 2018.

[16] A. A. Ansari, "Effect of Albedo on the motion of the infinitesimal body in circular restricted three-body problem with variable masses," Italian Journal of Pure and Applied Mathematics, vol. 38, pp. 581-600, 2017.

[17] A. A. Ansari, "Kind of Robe's restricted problem with heterogeneous irregular primary of N-layers when outer most layer has viscous fluid," New Astronomy, vol. 83, p. 101496, 2020.

[18] E. I. Abouelmagd, A. A. Ansari, and M. H. Shehata, "On Robe's restricted problem with a modified Newtonian potential," International Journal of Geometric Methods in Modern Physics, 2020.

[19] B. S. Kushvah, "Linear stability of equilibrium points in the generalized photogravitational Chermnykh's problem," Astrophysics and Space Science, vol. 318, no. 1-2, pp. 41-50, 2008.

[20] N. Pathak, V. O. Thomas, and E. I. Abouelmagd, "The perturbed photogravitational restricted three-body problem: analysis of resonant periodic orbits," Discrete and Continuous Dynamical Systems - Series S (DCDS-S), vol. 12, no. 4 and 5, pp. 849-875, 2019.

[21] N. Pathak, E. I. Abouelmagd, and V. O. Thomas, "On higher order of resonant periodic orbits in the photogravitational restricted three body problem," The Journal of the Astronautical Sciences, vol. 66, no. 4, pp. 475-505, 2019.

[22] A. A. Ansari and E. I. Abouelmagd, "Gravitational potential formulae between two bodies with finite dimensions,"
Astronomische Nachrichten, vol. 341, no. 6-7, pp. 656-668, 2020.

[23] B. Ishwar and A. Elipe, "Secular solutions at triangular equilibrium points in generalized photogravitational restricted three-body problem," Astrophysics and Space Science volume, vol. 277, pp. 437-446, 2001.

[24] J. Singh and J. J. Taura, "Motion in the generalized restricted three-body problem," Astrophysics and Space Science, vol. 343, no. 1, pp. 95-106, 2013.

[25] E. I. Abouelmagd and A. A. Ansari, "The motion properties of the infinitesimal body in the framework of bicircular Sun perturbed Earth-Moon system," New Astronomy, vol. 73, 2019.

[26] J. Singh and B. Ishwar, "Effect of perturbations on the stability of triangular points. In the restricted problem of three bodies with variable mass," Celestial Mechanics, vol. 35, no. 3, pp. 201-207, 1985.

[27] L. G. Lukyanov, "On the restricted circular conservative threebody problem with variable masses," Astronomy Letters, vol. 35, no. 5, pp. 349-359, 2009.

[28] E. I. Abouelmagd and A. Mostafa, "Out of plane equilibrium points locations and the forbidden movement regions in the restricted three-body problem with variable mass," Astrophysics and Space Science, vol. 357, no. 1, 2015.

[29] J. H. Jeans, Astronomy and Cosmogony, Cambridge University Press, Cambridge, England, 1928.

[30] M.-J. Zhang, C.-Y. Zhao, and Y.-Q. Xiong, "On the triangular libration points in photogravitational restricted three-body problem with variable mass," Astrophysics and Space Science, vol. 337, no. 1, pp. 107-113, 2012.

[31] I. V. Meshcherskii, Works on the Mechanics of Bodies of Variable Mass, GITTL, Moscow, Russia, 1949.

[32] M. Miyamoto and R. Nagai, "Three-dimensional models for the distribution of mass in galaxies," Publications of the Astronomical Society of Japan, vol. 27, pp. 533-543, 1975. 